\title{
Programmable retiming of an optical clock signal using the temporal Talbot effect
}

\author{
Santiago Tainta, María J. Erro, María J. Garde, and Miguel A. Muriel
}

\begin{abstract}
By using a Fourier processor based on the temporal Talbot effect and the properties of the Discrete Fourier Transform, we propose a scheme that adds an adjustable delay to a train of pulses while simultaneously preserving the clock recovery property of the Talbot effect. The obtained delay can be easily tuned by modifying only the electrical signal applied to an electro-optic modulator. Experimental results prove the validity of the proposal with a fine correspondence between the desired and measured delays all over a complete period of the signal.
\end{abstract}

Index Terms-Delay lines, Clock recovery, Fiber optics and optical communication, Talbot and self-imaging effects.

\section{INTRODUCTION}

Optical communications networks have greatly evolved in last years, with increasing functions assigned to the optical domain, such as time multiplexing or data routing. Among the tasks needed, an important one is phase synchronization or clock retiming, that is, adjusting the delay of an optical clock sequence to precisely adjust the position of the pulses within one period, so that i.e. optical time domain multiplexing can be achieved [1]. Usually a delay line is employed to fine tune the timing of the signal, existing several approaches mainly based on resonant elements with a slow-light effect or on the combination of wavelength dependent dispersion with wavelength converters [2-4]. Another alternative to generate time delays is to select a different and longer physical path for the signal [5]. In the last case, only a set of discrete values for the delay can be obtained, whereas for the other techniques the tuning can be continuous but usually slower and with a timebandwidth product limited by the characteristics of the used devices. To obtain a continuously tunable and wideband delay line more complex devices are required, such as the structure proposed in [6] where both approaches are combined.

In this work, we experimentally demonstrate the use of a simple all-optical setup that provides a continuously tunable delay all over the period of a clock signal formed by a train of optical pulses. Moreover, since the setup makes use of the temporal Talbot effect, it is able to recover a clock signal when some of the input pulses are missing, such as in the case of a Return-to-Zero (RZ) pulse sequence [7-10]. This combination

This paragraph of the first footnote will contain the date on which you submitted your paper for review. It will also contain support information, including sponsor and financial support acknowledgment.

This work was supported in part by the Ministerio de Economia y Competitividad of Spain under Grants TEC2014-58048-C2-1-P and TEC201458048-C2-2-P. of all optical clock recovery and retiming is achieved with only an electro-optic phase modulator and a dispersive element, avoiding thus some of the problems associated to other schemes, such as the requirement of high optical power, volume optics or high power losses in the setup.

\section{PROPOSED TECHNIQUE AND EXPERIMENTAL SETUP}

The proposed scheme is based on the use of an optical signal processor that enables the realization of the Discrete Fourier transform (DFT) of a repetitive sequence [11,12]. In such processor a periodic function, $c(t)$, modulates the phase and amplitude of an input train of pulses. If this signal is slow varying, the resulting modulation can be considered constant within the pulse duration and $c(t)$ is transformed into a periodic sequence of complex coefficients $c\left(t_{n}\right)=c_{n}$. Then, using a specific case of the fractional temporal Talbot effect [13] as further specified below, it was demonstrated in [11] that the relationship between the pulses' amplitudes at the output, $C_{k}$, and the applied complex coefficients, $c_{n}$, is determined by the DFT, allowing the Fourier synthesis of the applied sequence as a function of the desired output. Thus, if the electric field at the output of the modulating stage is given by:

$$
\mathrm{E}(t) \propto \sum_{n=-\infty}^{\infty} c_{n} \cdot a\left(t-n T_{0}\right)
$$

then the power at the output of such system can be expressed as [11,12]:

$$
P_{\text {out }}(t) \propto \frac{1}{N^{2}} \sum_{k=-\infty}^{\infty}\left|C_{k}\right|^{2}\left|a\left(t+k \frac{T_{0}}{N}-\frac{T_{0}}{2}\right)\right|^{2}
$$

where $T_{0}$ is the temporal separation between two adjacent pulses in the input train of pulses, $a(t)$ corresponds to the shape of the input optical pulses with a pulse-width determined by $\Delta t$, $N$ corresponds to the period of the sequence $c_{n}$, and the modulation signal period is $N \cdot T_{0}$. In order for the optical processor to operate properly the system must fulfil the two conditions presented in [11,12]. First, in order to avoid the

S. Tainta, MJ. Erro and MJ. Garde are with the Electrical, Electronics and Communications Engineering Department, Public University of Navarra, 31006 Pamplona, Spain (corresponding author: santiago.tainta@unavarra.es)

M.A Muriel is with the Department of Photonic Technology, Universidad Politécnica de Madrid, 28040 Madrid, Spain. 
overlapping of pulses at the output of the optical processor, the pulse width has to verify $\Delta t<T_{0} / N$. Second, equation (2) was obtained in [11] when the dispersive device produces a fractional Talbot case and the coprime integers $s$ and $m$ defined in [13] have the values $s=1$ and $m=N^{2}$. This corresponds to a dispersion value given by $\ddot{\varphi}=\left(N T_{0}\right)^{2} / 2 \pi N^{2}=T_{0}^{2} / 2 \pi$ $[11,12]$. Besides, it is worth emphasizing that, from equation (2), a temporal scaling factor is present at the output train of pulses. This additional scaling results in the presence of the complete transformed sequence within a single period of the original train.

In our case, the processing is based on the well-known shift theorem of the DFT, which states that, when a linear phase modulation is applied in either domain, the sequence in the transformed domain suffers a circular shift. When applying this property to an all-ones sequence, it results in:

$$
\begin{gathered}
c_{n}=\left[1 \cdot \exp \left(j 2 \pi \frac{L}{N} n\right)\right] \stackrel{D F T}{\longrightarrow} \\
C_{k}= \begin{cases}N & \text { if } k=L, L \pm N, L \pm 2 N, \ldots \\
0 & \text { otherwise }\end{cases}
\end{gathered}
$$

where $L$ corresponds to an arbitrary integer that controls the shifting produced at the output. For simplicity, we will choose $L$ to verify that $L / N$ is an irreducible fraction. By combining equations (2) and (3), the output power of the system when the input train of pulses is modulated with such coefficients can be obtained:

$$
P_{\text {out }}(t) \propto \sum_{k=-\infty}^{\infty}\left|a\left(t-k T_{0}+\frac{L}{N} T_{0}-\frac{T_{0}}{2}\right)\right|^{2}
$$

As indicated by equation (4), the input pulse train has been time-shifted by half a period. Also, an additional delay $\tau=T_{0} L / N$ is present at the output whose value is determined by the relationship between $L$ and $N$. It is worth emphasizing that, as $L$ and $N$ can be arbitrarily chosen without any variation on the introduced dispersion, any fractional delay can be attained within the period $T_{0}$ without modifying the required setup.

The previous equations were derived when the input sequence of pulses has uniform amplitude, that is, when it is an all-ones sequence. However, as proved in previous proposals [7-10], the dispersion in our setup also acts as a clock recovery system due to the distributed interference of several pulses when an aperiodic pulse train is introduced at the input. Consequently, if a RZ data-modulated pulse train is passed through the proposed retiming scheme, the final output train will be a recovered uniform train of pulses, that is, a clock signal where a tunable time delay has been introduced by adequately selecting the electric signal applied to the modulator.

The setup shown schematically in Fig. 1 was used to perform a proof-of-concept experiment. An active mode-locked laser that generates nearly transform-limited pulses was used as optical source. It was tuned to produce 4 ps-width pulses with a repetition rate of $9.881 \mathrm{GHz}$ at a central wavelength of
$1541.5 \mathrm{~nm}$. The generated train of pulses was intensity modulated using a $\mathrm{LiNbO}_{3}$ Mach-Zehnder modulator to which a random binary sequence (RBS) was applied to generate a RZ data signal. A limit on the acceptable number of consecutive zeros in the data signal was introduced due to the limitations that this parameter imposes on clock recovery setups based on the temporal self-imaging effect $[7,8]$, as will be commented later. The data modulated signal was passed to the retiming scheme, which consists on an electro-optic phase modulator and a dispersive medium. The phase modulator was driven with an Arbitrary Waveform Generator (AWG) with 10 bits resolution and $12.5 \mathrm{GHz}$ bandwidth that generated the required signal to control the retiming. Afterwards, a Linearly Chirped Fiber Bragg Grating (LC-FBG) was used as the dispersive medium with a second order dispersion of approximately $-1630 \mathrm{ps}^{2}$, which corresponds to the required temporal selfimaging case as determined by [11,12], all over a $7 \mathrm{~nm}$ bandwidth. The signals at the input and at the output of the setup were analyzed using the combination of a photodiode and a sampling scope with a $65 \mathrm{GHz}$ bandwidth. Additionally, the electrical spectrum of the signals was measured using a $30 \mathrm{GHz}$ photodetector and an Electrical Spectrum Analyzer (ESA).

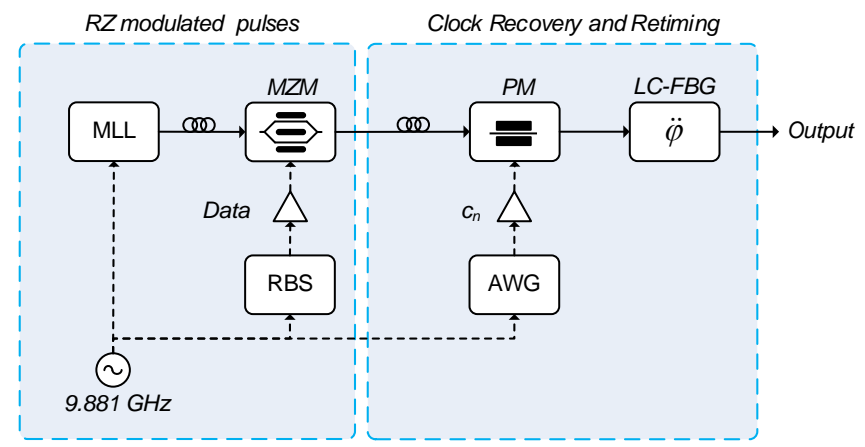

Fig. 1. System setup (MLL: Mode-Locked Laser, RBS: Random Binary Sequence, MZM: Mach-Zehnder Modulator, PM: Phase Modulator, AWG: Arbitray Waveform Generator, LC-FBG: Linearly Chirped-Fiber Bragg Grating)

To induce a specific delay on the output train, $\tau$, a phase profile as determined by equation (3) has to be used, with the values of $L$ and $N$ calculated as the numerator and denominator of the irreducible fraction that verifies $L / N=\tau / T_{0}$. In order to reduce the requirements that generating such a signal would impose on the AWG, we take advantage of the intrinsic periodicity of the phase, which allows the clamping of the required phase coefficients between $-\pi$ and $\pi$. Some examples of such modulating signals can be observed in Fig. 2, where the measured voltages generated by the AWG are shown along with the ideal voltage patterns for $N=2,4$ and 16 and $L=1$. An amplification stage that introduced a $26 \mathrm{~dB}$ gain was required just between the AWG and the modulator to ensure that the modulating voltage varied between $5 \mathrm{~V}$ and $-5 \mathrm{~V}$, as the half wave voltage on the used phase modulator was $V_{\pi}=5 \mathrm{~V}$. As it can be observed, the obtained voltages are only limited by the resolution of the employed AWG, being this the principal limiting factor for the attainable resolution in the resulting delay. Also, as the AWG must operate at the same rate as the input train of pulses, that is, 9.881 GSps, the operation 
bandwidth of the electric components should be large enough in order to avoid distorting the signal. In our case, this problem is not present as all the components used (AWG, phase modulator and driver) have a bandwidth of at least $12.5 \mathrm{GHz}$, but it is a factor that might also limit the attainable delays if not taken into consideration.

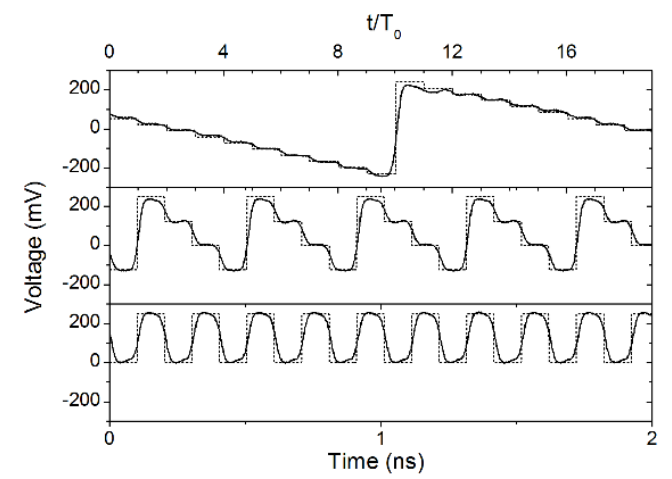

Fig. 2: Ideal (dashed) and measured (black) voltage phase profiles delivered by the AWG for $L=1$ and $N=16$ (top), 4 (mid) and 2 (bottom) which correspond to a delay at the output of $T_{0} / 16, T_{0} / 4$ and $T_{0} / 2$ respectively

\section{EXPERIMENTAL RESULTS AND DISCUSSIONS}

Fig. 3 shows the measured signals at the input and output of the setup when no signal is applied to the phase modulator (no retiming induced in the setup) and a maximum of five consecutive zeros in the RBS data is allowed. A clear baseline can be observed in the eye diagram of the input RZ-OOK data signal shown in Fig. 3a, revealing an intermittent train that contains both " 1 "-bits with a pulse in the center of the period and "0"-bits without any pulse within the period. Correspondingly, the RF spectrum (Fig. 3b) encompasses the clock component at a frequency of $9.881 \mathrm{GHz}$ and a noise-like spectral background component due to the randomly varying modulating signal. Fig. 3c and 3d present the eye diagram and RF spectrum at the output. Now, neglecting the vicinity of the clock frequency, the measured RF spectrum shows a suppression of $\sim 9 \mathrm{~dB}$ of the modulated data, confirming the filtering effect of the temporal Talbot effect. The recovered clock signal is visible in the eye diagram of Fig. 3c, although its quality is degraded due to strong amplitude fluctuations. These amplitude fluctuations are inherent to clock recovery setups based on the temporal self-imaging effect [7-10] and a more precise adjustment of the applied dispersion would help reducing them $[7,14]$. However, in order to suppress them, the addition of other components, such as a SOA in conjunction with an amplitude limiter, would be required [10].

In order to quantify the pulse to pulse amplitude variation, we use the Coefficient of Variation (CV) which is defined as the ratio of the standard deviation to the mean peak power of the output pulses. In our setup, the input data signal presents a value of 0.07 while the recovered clock in Fig. 3c has a CV of 0.46 . The increased smearing of the peak power is explained by the fact that the recovered output pulses result from the interference of several dispersed pulses, so that the final peak level depends on the number of interfering pulses that were present in the original random pulse train. With narrower input pulses or higher dispersion values, the result is a higher number of adjacent bits that, once dispersed, have a significant contribution in the formation of the pulse within one specific bit, thus reducing the $\mathrm{CV}$. When those values are fixed, as in our case, the CV only varies with the probability that in the contributing adjacent bits a pulse was indeed present, which only occurs for a " 1 ” pulse in the RZ data stream [7,8]. In effect, our experimental results show that the CV suffers a larger deterioration as the number of zeros is increased. This is shown, in Fig. 4, where the CV of the output pulses peaks is shown as a function of the maximum allowed number of consecutive zeros for the different delays shown in Fig. 5, along with the mean value in each case. Also, there is an additional constant degradation of the CV due to the imperfections of the LC-FBG used [14], which correspond to the ripple present in the delay, the offset between its dispersion and the ideal Talbot dispersion that in our case is around a $0.45 \%$, and the limited bandwidth. The most remarkable fact is, however, that the CVs values do not differ significantly when different phase profiles are applied to the input train of pulses, revealing that the retiming of the output signal does not affect the clock recovery capabilities of the setup or viceversa. It is worth mentioning that, in the case of a maximum of ten consecutive zeros, a large dispersion of the obtained CV values for the different delays was observed, which is attributed to an instability in the measurement setup that occurred in that specific measurement.
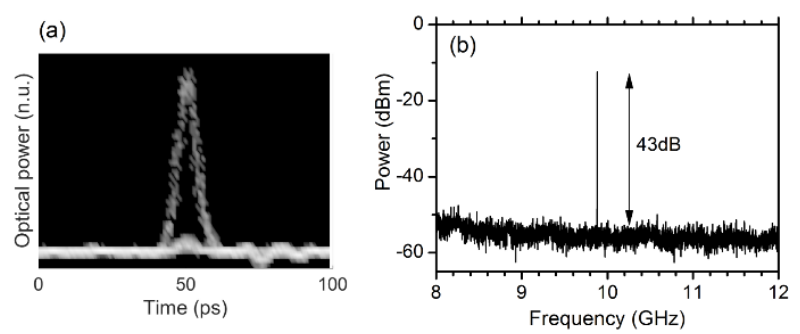

(c)
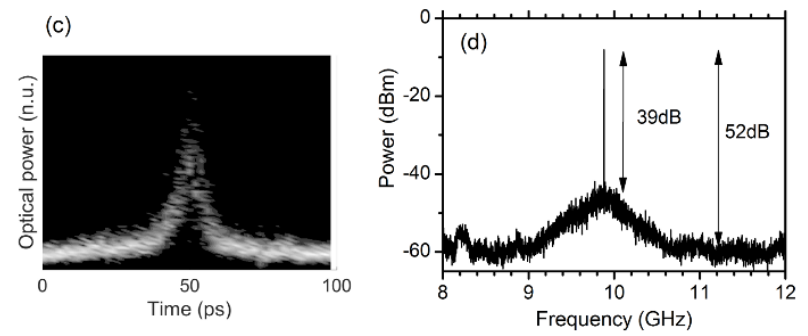

Fig. 3: Eye diagram and electrical spectrum of the RBS modulated signal at the input (a, b) of the clock retiming and recovery scheme and at the output (c, d)

Finally, Fig. 5 shows the clock retiming capabilities of the proposed setup by comparing the expected theoretical delay for a determined set of coefficients with the experimentally obtained values, for different numbers of maximum consecutive zeros allowed. To verify the tunability of the system, 16 different delays evenly spaced along $T_{0}$ were selected, establishing for each case the parameters $L$ and $N$ as those that form an irreducible fraction which corresponds to the desired delay and applying the resulting phase profiles generated with the phase modulator. For each sequence the position of the output pulses with and without activating the 
signal from the AWG are compared in the oscilloscope using an external trigger source to determine the obtained delay. This process is repeated 500 times and the mean and standard deviation of these measurements are represented in Fig. 5. As it can be observed, the measured results are in very good agreement with the expected values, with a coefficient of determination above 0.98 in all the cases when doing the linear regression of the obtained data. Also, the resulting variance in the experimental results is quite consistent among all the cases, with a value below $3 \mathrm{ps}$, which is within the error range of our measurement setup, except in the case of ten consecutive zeros maximum (Fig. 5e) which is attributed to the previously mentioned experimental problem. Thus, the system can operate even when the number of zeros is increased, although this will increase the peak amplitude variance of the recovered clock. In all cases, with the appropriate electrical signals, the delay can be adjusted over an entire period ( 101 ps), allowing the complete retiming of the recovered clock, without incurring in extra penalties.

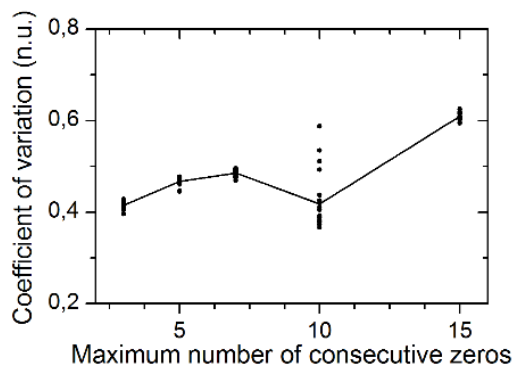

Fig. 4. Coefficient of Variation as a function of the number of consecutive zeros in the modulated data (dots) and mean value (solid line).
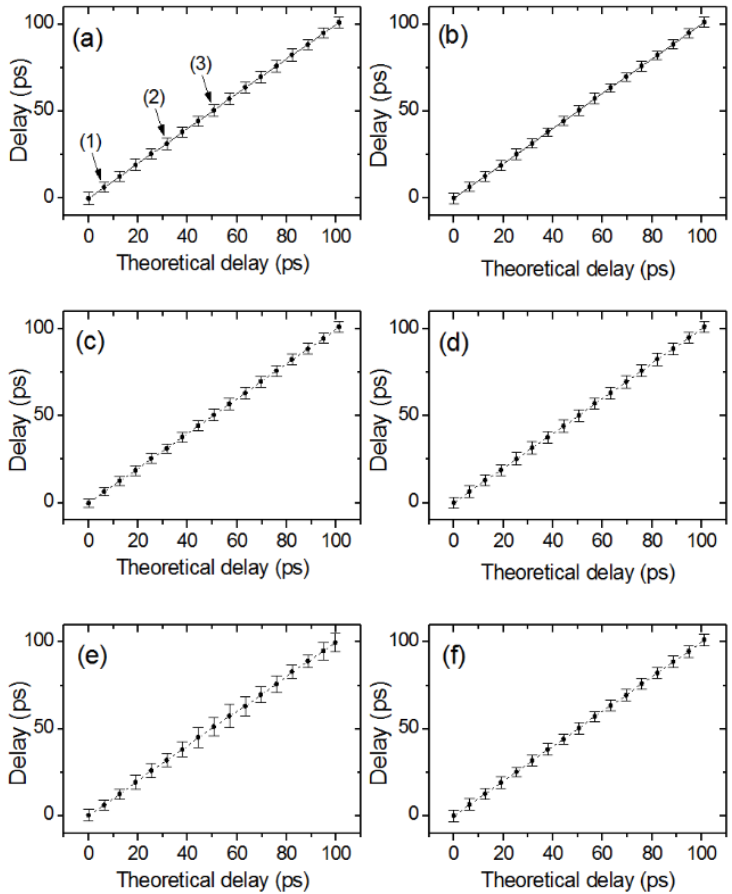

Fig. 5: Measured delay at the output compared to the theoretical delay when no data are applied (a) and when data are applied but the maximum number of consecutive zeros is limited to 3 (b), 6 (c), 7 (d), 10 (e) and 15 (f). (1), (2) and (3) correspond to the delays obtained when applying the phase profiles in Fig.
2 top, middle and bottom respectively.

\section{CONCLUSIONS}

To sum up, we have proposed and experimentally demonstrated a simple scheme based on all-fiber technology for the recovery and retiming of an optical clock signal. The system is based on the combined use of the properties of the DFT and the temporal Talbot effect. It has been proven that the setup allows the tunable control of the timing at the output clock all over a complete clock period, just by modifying the phase profile generated by the AWG and without changing the dispersive medium. Moreover, we have verified that the retiming capability of the scheme is valid even when a decreasing density of ones is present in the input signal. However, in that case the recovered clock pulses will exhibit amplitude variations intrinsic to the use of the temporal self-imaging effect for clock recovery that will degrade the performance of the system.

\section{REFERENCES}

[1] M. Lévesque and D. Tipper, "A Survey of Clock Synchronization Over Packet-Switched Networks,” IEEE Comm. Surveys \& Tutorials, vol. 18, no. 4, pp. 2926-2947, Fourth Quarter 2016.

[2] "Delay lines," H. Shahoei and J.P. Yao, Wiley Encyclopedia of Electrical and Electronics Engineering, 1-15, (2014).

[3] A. Melloni et al., "Tunable delay lines in silicon photonics: coupled resonators and photonic crystals, a comparison,” IEEE Photonics J, vol. 2, no. 2, pp. 181-194, Apr. 2010. DOI: 10.1109/JPHOT.2010.2044989.

[4] F. Parmigiani et al., " All-optical pulse reshaping and retiming systems incorporating pulse shaping fibre Bragg gratings," J. Lightw. Technol., vol. 24, no. 1, pp. 357-364, Jan. 2006.

[5] K. Jinguji et al., "Two-port optical wavelength circuits composed of cascaded Mach-Zehnder interferometers with point-symmetrical configurations,” J Lightwave Technol, vol. 14, no. 10, pp. 2301-2310, Oct. 1996. DOI: $10.1109 / 50.541222$

[6] X. Wang et al., "Continuously tunable ultra-thin silicon waveguide optical delay line,” Optica, vol. 4, no. 5, pp. 507-514, May 2017. DOI: 10.1364/OPTICA.4.000507

[7] D. Pudo et al., "Single and multiwavelength all-optical clock recovery in single-mode fiber using the temporal Talbot effect," J. Lightw. Technol., vol. 25, no.10, pp. 2898-2903, Oct. 2007.

[8] R. Maram et al., "Sub-harmonic periodic pulse train recovery from aperiodic optical pulse sequences through dispersion-induced temporal self-imaging," Opt Express, vol. 23, no. 3, pp. 3602-3613, 9 Feb. 2015. DOI: 10.1364/OE.23.003602

[9] J. Jeon et al., "Programmable sub-harmonic optical clock recovery based on dispersion-induced inverse temporal self-imaging," 2018 Conference on Lasers and Electro-Optics (CLEO), San Jose, CA, 2018, pp. 1-2.

[10] M. Oiwa et al., "Temporal-Talbot-effect-based preprocessing for pattern-effect reduction in all-optical clock recovery using a semiconductor-optical-amplifier-based fiber ring laser," Optical Fiber Technology, vol. 16, no. 1, pp. 63-71, Jan. 2010. DOI: 10.1016/j.yofte.2009.10.004

[11] S. Tainta et al., "Temporal self-imaging effect for periodically modulated trains of pulses," Opt Express, vol. 22, no. 12, pp. 1525115266, 16 Jun. 2014. DOI: 10.1364/OE.22.015251

[12] C.R. Fernández-Pousa, "A dispersion-balanced Discrete Fourier Transform of repetitive pulse sequences using temporal Talbot effect," Optics Communications, vol. 402, pp. 97-103, 1 Nov. 2017. DOI: 10.1016/j.optcom.2017.05.071

[13] J. Azaña and M.A. Muriel, "Temporal Talbot effect in fiber gratings and its applications,” Appl Opt, vol. 38, no. 32, pp. 6700-6704, Nov. 1999. DOI: $10.1364 / A O .38 .006700$

[14] M. Oiwa et al., "Influence of nonideal chirped fiber Bragg grating characteristics on all-optical clock recovery based on the temporal Talbot effect,” Appl Opt, vol. 48, no. 4, pp. 679-690, 1 Feb. 2009. DOI: 10.1364/AO.48.000679 